\title{
Quantitative Assessment of Proprioception Using Dynamometer in Incomplete Spinal Cord Injury Patients: A Preliminary Study
}

\author{
Won Kee Chang, MD, Yun Sulk Jung, MD, Mi-Kyoung Oh, MD, Keewon Kim MD, MS
}

Department of Rehabilitation Medicine, Seoul National University Hospital, Seoul, Korea

\begin{abstract}
Objective To investigate the feasibility of a knee proprioception evaluation using a dynamometer as a tool for evaluating proprioception of the lower extremities in patients with incomplete spinal cord injury (SCI), and to explore its usefulness in predicting the ambulatory outcome.

Methods A total of 14 SCI patients (10 tetraplegic, 4 paraplegic; all AIS D) were included in this study. The passive repositioning error (PRE) and active repositioning error (ARE) were measured with a dynamometer, along with tibial somatosensory evoked potential (SSEP) and abductor hallucis motor-evoked potential (MEP). Ambulatory capacity was assessed with the Walking Index for Spinal Cord Injury II (WISCI-II), both at the time of the proprioception test (WISCI_i) and at least 6 months after the test (WISCI_6mo).

Results The PRE showed a negative correlation with WISCI_i $(\mathrm{r}=-0.440, \mathrm{p}=0.034)$ and WISCI_6mo $(\mathrm{r}=-0.568$, $\mathrm{p}=0.010)$. Linear multiple regression showed the type of injury, lower extremities motor score, MEP, and PRE accounted for $75.4 \%$ of the WISCI_6mo variance $(\mathrm{p}=0.080)$.

Conclusion Proprioception of the knee can be measured quantitatively with a dynamometer in patients with incomplete SCI, and PRE was related to the outcome of the ambulatory capacity. Along with the neurological and electrophysiological examinations, a proprioception test using a dynamometer may have supplementary value in predicting the ambulatory capacity in patients with incomplete SCI.
\end{abstract}

Keywords Spinal cord injuries, Proprioception, Walking

\section{INTRODUCTION}

Predicting ambulatory capacity is critical in patients with spinal cord injury (SCI). Clinical and electrophysi- ological examinations are used to assess the degree of neurological deficit, the level of injury, and the prognosis of patients with SCI [1-5]. In clinical examinations, the American Spinal Injury Association (ASIA)/International

Received June 7, 2016; Accepted August 31, 2016

Corresponding author: Keewon Kim

Department of Rehabilitation Medicine, Seoul National University Hospital, Seoul National University College of Medicine, 101 Daehak-ro, Jongno-gu, Seoul 03080, Korea. Tel: +82-2-2082-2619, Fax: +82-2-743-7473, E-mail: keewonkimm.d@gmail.com

ORCID: Won Kee Chang (http://orcid.org/0000-0001-9756-6817); Yun Suk Jung (http://orcid.org/0000-0001-6683-9499); Mi-Kyoung Oh (http://orcid. org/0000-0002-2900-7156); Keewon Kim (http://orcid.org/0000-0001-6597-578X).

@ This is an open-access article distributed under the terms of the Creative Commons Attribution Non-Commercial License (http://creativecommons.org/ licenses/by-nc/4.0) which permits unrestricted noncommercial use, distribution, and reproduction in any medium, provided the original work is properly cited. Copyright (c) 2017 by Korean Academy of Rehabilitation Medicine 
Spinal Cord Society (ISCoS) neurological standard scale (AIS) is widely used by clinicians in evaluating the degree of SCI and predicting the functional outcome in patients $[6,7]$. Electrophysiological examinations, including the tibial somatosensory evoked potential (SSEP) and lowerextremity motor-evoked potential (MEP), supplement the clinical examination in predicting the ambulatory capacity in SCI patients $[1,2,8]$.

In the clinical setting, proprioception of patients with SCI is measured along with the physical examination and SSEP. However, one of the major drawbacks in assessing proprioception with these methods is that the results cannot be interpreted in a quantitative manner; the physical examination offers an all-or-none information, and SSEP results are interpreted in categorized groups.

Currently, a knee proprioception test using a dynamometer is performed in the orthopedic field for measuring the knee proprioception of patients with osteoarthritis or anterior cruciate ligament injuries $[9,10]$. The test has an advantage of providing quantitative measurement of knee proprioception by the angle of error in knee joint repositioning, and its reliability and validity have been proved in previous studies $[11,12]$.

In this study, we used the dynamometer in incomplete SCI patients, and assessed the feasibility of the knee proprioception test as a tool for evaluating lower-extremity proprioception, and its clinical usefulness as a supple- ment in predicting the ambulatory outcome.

\section{MATERIALS AND METHODS}

\section{Participants}

A total of 14 patients with acute to subacute incomplete SCI, who underwent a knee proprioception test using a dynamometer from January 2013 to September 2015 at Seoul National University Hospital, were reviewed retrospectively. Patients with a history of knee injury or surgery, peripheral polyneuropathy, a neuromuscular disorder, a cerebral lesion, or a psychiatric/cognitive disorder were excluded. This study was approved by the Institutional Review Board of Seoul National University Hospital (1604-053-753).

The clinical characteristics of patients are shown in Table 1. Fourteen patients ( 8 men, 6 women) with incomplete SCI were included in the study. The mean age was $62.7 \pm 10.6$ years, and the average time since injury (TSI) was $4.7 \pm 2.3$ weeks. All patients were classified as AIS D; 10 patients had incomplete tetraplegia, while 4 patients had paraplegia. No significant differences were observed in the lower extremities motor score (LEMS) or ambulatory capacity between patients with tetraplegia and paraplegia. (LEMS 43.30 vs. 37.25 , p=0.142; WISCI_i 14.60 vs. $13.00, \mathrm{p}=0.488)$.

Table 1. Characteristics of subjects with incomplete spinal cord injury $(\mathrm{n}=14)$

\begin{tabular}{cccclcl}
\hline ID & Age (yr) & Sex & AIS & NLI & TSI (wk) & \multicolumn{1}{c}{ Cause of injury } \\
\hline 1 & 55 & M & D & C4 & 8 & Spinal hemangioma \\
\hline 2 & 36 & F & D & T12 & 2 & TB spondylitis \\
\hline 3 & 70 & M & D & C6 & 9 & Ependymoma \\
4 & 73 & M & D & C6 & 4 & CSM \\
\hline 5 & 55 & F & D & C4 & 4 & Traumatic \\
6 & 72 & M & D & C4 & 8 & Chondroma \\
\hline 7 & 67 & F & D & C5 & 4 & CSM \\
\hline 8 & 70 & F & D & C4 & 6 & CSM \\
\hline 9 & 53 & F & D & L3 & 1 & Syringomyelia \\
10 & 63 & M & D & L2 & 4 & Spinal infarction \\
11 & 68 & M & D & L2 & 4 & Aspergilloma \\
\hline 12 & 56 & F & D & C4 & 2 & Traumatic \\
\hline 13 & 65 & M & D & C5 & 6 & CSM \\
\hline 14 & 75 & M & D & C4 & 4 & CSM \\
\hline
\end{tabular}

ID, identification; AIS, American Spinal Injury Association neurologic standard scale; NLI, neurological level of injury; TSI, time since injury; TB, tuberculosis; CSM, cervical spondylotic myelopathy. 


\section{Knee proprioception assessment}

A dynamometer (System 4 Pro; Biodex Medical Inc., Shirley, NY, USA) was used to evaluate the knee proprioception. Patients were seated in a chair with their knee and hip positioned at $90^{\circ}$ of flexion. The ankle was cuffed with a strap and the foot was rested in neutral position. The posture was stabilized with chest, hip, and midthigh straps, as shown in Fig. 1. Patients were blindfolded throughout the examination, and were directed by the examiner.

Knee proprioception was measured in both active and passive positions by the degree of repositioning error. The active repositioning sense was evaluated as follows: the knee joint of the patient was placed at a specific angle for 10 seconds, and they were instructed to memorize the joint position sense. Starting from the $90^{\circ}$ flexion, patients were told to reposition their knee joint to reproduce the predetermined angle. The degree of error between the reproduced angle and the pre-designated angle of knee flexion was defined as the active repositioning error (ARE). The passive repositioning sense was evaluated with passive extension of the knee joint by the dynamometer at a speed of $2^{\circ}$ per second, and the patients were instructed to say "stop" when the knee joint angle reached the predetermined angle. The degree of error between the reproduced angle and the pre-designated knee flexion angle was defined as the passive repositioning error

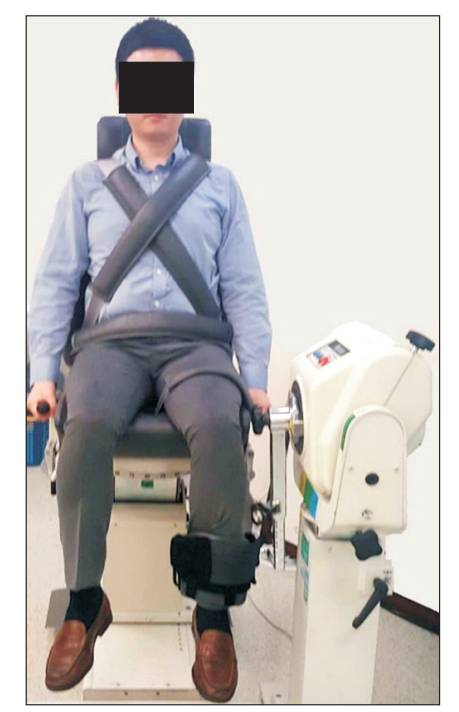

Fig. 1. Patient positioned in dynamometer for left knee proprioception test (System 4 Pro; Biodex Medical Inc., Shirley, NY, USA).
(PRE). The test was performed at knee flexion of $30^{\circ}$ and $60^{\circ}$ bilaterally, three times each.

\section{Neurologic and electrophysiological examinations}

A neurologic examination was conducted in patients with incomplete SCI, as per the International Standards for Neurological Classification of Spinal Cord Injury (ISNCSCI) [13]. The LEMS was defined as the sum of muscle function grading in the 5 key muscles of the bilateral lower extremities (0-50).

For electrophysiological examinations, we performed the tibial SSEP and abductor hallucis (AH) MEP. Tibial SSEP values were elicited by electrical stimulation of the tibial nerves at the medial ankle. Scalp electrodes were positioned at $\mathrm{Cz}^{\prime}-\mathrm{Fz}$ (International 10/20 electrode system). The P40 latency and amplitude were recorded and classified into three categories: Category 1 , no SSEP recording; Category 2, pathologic P40 latency or amplitude; Category 3, normal P40 latency and amplitude (cutoff value: P40 latency, $41.3 \mathrm{~ms}$; amplitude, $1.4 \mu \mathrm{V}$ ) [14].

MEP were elicited with transcranial magnetic stimulation using a cone-shaped coil positioned tangentially to the scalp over Cz. Recordings of MEP were obtained with surface electrodes bilaterally attached to the belly of the AH. Motor conduction and F-wave latency were examined at the bilateral tibial nerve at the $\mathrm{AH}$, and the central motor conduction time (CMCT) was calculated using the following formula: MEP latency- $\{(\mathrm{F}$ wave latency $+\mathrm{M}$ wave latency-1) $\times 0.5\}$. MEP results were classified into three categories: Category 1, no MEP recording; Category 2, pathologic CMCT latency; Category 3, normal CMCT latency (cutoff value: CMCT, $15.9 \mathrm{~ms}$ ) [15].

\section{Ambulatory capacity}

Ambulatory capacity was assessed with the Walking Index for Spinal Cord Injury II (WISCI-II), which incorporates a numeric scale of 0 to 20 for evaluating the ambulatory capacity of SCI patients. A higher score represents better ambulatory function of the patient: 0 indicates the patient is unable to stand or participate in walking, while 20 indicates the patient can ambulate with no devices, no braces and no physical assistance [16]. The WISCI-II was measured at least twice in a patient; the initial WISCI (WISCI_i) was performed at the time of the neurological/electrophysiological examination, and the follow-up WISCI was performed at least 6 months after the initial 

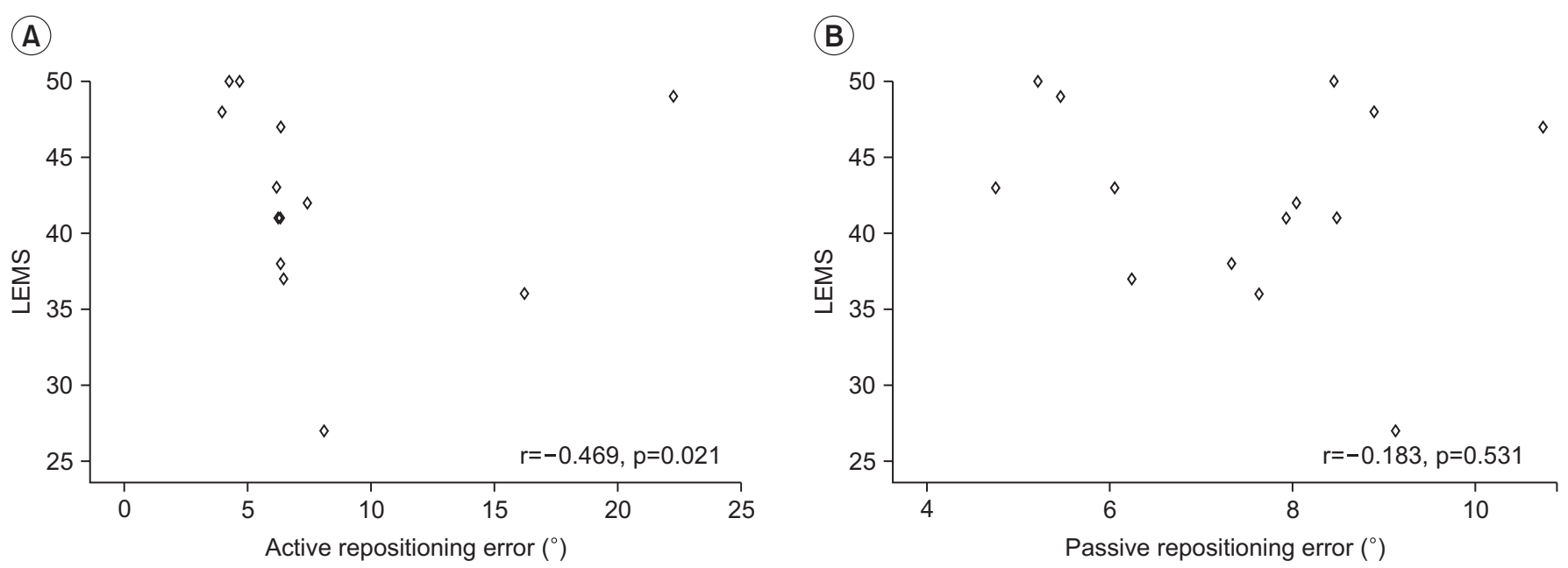

Fig. 2. Correlation between the repositioning error and lower extremities motor power. (A) Active repositioning error and (B) passive repositioning error. LEMS, lower extremities motor score.

examination(WISCI_6mo).

\section{Statistical analysis}

IBM SPSS ver. 21.0 (IBM Corp., Armonk, NY, USA) was used for statistical analysis. Spearman correlation analysis evaluated the relationship between ambulatory capacity and clinical variables, including knee proprioception measured with a dynamometer. The Kruskal-Wallis test assessed whether there were significant differences in ambulatory capacity among the SSEP and MEP categories. Linear multiple regression analysis was performed to compare the explanatory power of PRE and SSEP as proprioceptive parameters in predicting WISCI_6mo. Regression models were established with demographic (age, TSI, type of injury, and sex) and clinical parameters, in addition to proprioception parameter (PRE or SSEP), to find a model having the strongest explanatory powers.

\section{RESULTS}

Knee proprioception measured with a dynamometer

The average PRE was $7.44^{\circ} \pm 1.65^{\circ}$, and the average ARE was $7.93^{\circ} \pm 4.87^{\circ}$. ARE had a negative correlation with LEMS $(\mathrm{r}=-0.469, \mathrm{p}=0.021)$ (Fig. 2). No other significant correlations were observed between clinical/neurological and proprioception parameters. Table 2 shows the clinical, neurological, electrophysiological, and proprioception data of the 14 participants.
Table 2. Clinical, neurological, electrophysiological, and proprioception parameters of patients with spinal cord injury

\begin{tabular}{|cc}
\hline \multicolumn{1}{c}{ Parameter } & Value \\
\hline Age $(\mathrm{yr})$ & $62.7 \pm 10.6(36-75)$ \\
\hline Sex (male:female) & $8: 6$ \\
\hline TSI (wk) & $4.7 \pm 2.3(1-9)$ \\
\hline LEMS & $42.3 \pm 6.2(28-50)$ \\
\hline SSEP category & \\
\hline 1 & 4 \\
\hline 2 & 3 \\
\hline 3 & \\
\hline MEP category & 2 \\
\hline 1 & 3 \\
\hline 2 & 3 \\
\hline 3 & $7.93 \pm 4.87(3.97-22.28)$ \\
\hline ARE $\left({ }^{\circ}\right)$ & $7.44 \pm 1.65(4.74-10.75)$ \\
\hline PRE $\left({ }^{\circ}\right)$ & \\
\hline
\end{tabular}

Values are presented as mean \pm standard deviation (range). TSI, time since injury; LEMS, lower extremities motor score; SSEP, somatosensory evoked potential; MEP, motor-evoked potential; ARE, active repositioning error; PRE, passive repositioning error.

Relationship between ambulatory capacity and clinical/ electrophysiological examination scores

WISCI_i positively correlated with LEMS $(\mathrm{r}=0.447$, $\mathrm{p}=0.033$ ), while it was negatively correlated to PRE ( $\mathrm{r}=$ $-0.440, p=0.034)$. WISCI_6mo had a negative correlation with PRE ( $r=-0.568, p=0.010)$. No other significant cor- 
relations existed between the ambulatory capacity and clinical/proprioception parameters (Table 3).

The tibial SSEP results were allocated into three categories, based on the P40 latency and amplitude. No significant differences were seen in the ambulatory capacity among the three categories (WISCI_i $\mathrm{p}=0.750$; WISCI_6mo $\mathrm{p}=0.651)$. The AH MEP scores were also allocated into three categories according to the CMCT, and there were no significant differences in ambulatory capacity among the groups (WISCI_i $\mathrm{p}=0.349$; WISCI_6mo $\mathrm{p}=0.642$ ) (Table 4).

\section{Linear multiple regression model for predicting} ambulatory capacity after 6 months (PRE vs. SSEP)

Table 5 shows the multiple regression analysis on WISCI_6mo in response to demographic and clinical/ proprioception parameters. The regression model with type of injury (tetraplegia or paraplegia), LEMS, MEP, and PRE, accounted for totally $75.4 \%$ of the WISCI_6mo variance $(\mathrm{p}=0.080)$, showing the strongest explanatory power.

\section{DISCUSSION}

Knee proprioception is perceived by the mechanoreceptors in the joint capsule and ligamentous insertions of the knee joint [17], and is relayed to the cerebral cortex via the dorsal column-medial lemniscus pathway. This proprioception feedback is important in generating anticipatory movement during the gait cycle [18], and the impairment of the lower-extremity proprioception in SCI

Table 3. Correlation between WISCI and clinical parameters, including knee proprioception

\begin{tabular}{ccccc}
\hline Parameter & Age & LEMS & PRE & ARE \\
\hline WISCI_i & & & & \\
r & -0.351 & $0.477^{*}$ & $-0.440^{*}$ & -0.139 \\
p-value & 0.093 & 0.033 & 0.034 & 0.503 \\
WISCI_6mo & & & & \\
r & -0.369 & 0.289 & $-0.568^{*}$ & -0.108 \\
p-value & 0.096 & 0.195 & 0.010 & 0.623 \\
\hline
\end{tabular}

WISCI, Walking Index for Spinal Cord Injury; WISCI_i, WISCI-II initial; WICSI_6mo, WISCI-II after 6 months; LEMS, lower extremities motor score; PRE, passive repositioning error; ARE, active repositioning error. ${ }^{*} \mathrm{p}<0.05$. patients reduces the ability to organize an optimal movement pattern while walking [19].

In this study, we measured the patients' knee proprioception, by both active and passive positioning of the knee, using a dynamometer. Proprioception assessment by passive positioning is measured, regardless of the patients' lower-extremity motor power, while active positioning requires sufficient knee flexor muscle power to reproduce the angle. This explains the negative correlation between ARE and LEMS. Therefore, we hypothesize that PRE better reflects the extent of proprioception impairment than ARE.

Since our study did not contain a control group, a direct comparison of knee proprioception between SCI patients and non-SCI control subjects was not possible. However, comparing the PRE measured from our study with previously published studies of the knee proprioception test administered to normal subjects, which range from $2.43^{\circ}$ to $5.90^{\circ}$ [20-22], we assume the SCI patients in our study have impairment of knee proprioception. To confirm our assumption, further studies are required, which compares both control group and SCI group head-on.

In assessing the long-term ambulatory outcome of SCI patients, the lower-extremity SSEP and MEP are considered to be useful tools. However, in this study, no significant differences in ambulatory capacity between groups allocated by the SSEP and MEP were observed. The small number of subjects in our study may be one of the reasons for this result.

LEMS is positively correlated with WISCI at the time of

Table 4. Kruskal-Wallis test for ambulatory capacity of SSEP and MEP categories

\begin{tabular}{lcccc}
\hline & WISCI_i & p-value & WISCI_6mo & p-value \\
\hline SSEP category & & 0.750 & & 0.651 \\
1 & 12.75 & & 19.50 & \\
2 & 12.00 & & 18.00 & \\
3 & 15.67 & & 19.00 & \\
MEP category & & 0.349 & & 0.642 \\
1 & 8.50 & & 19.50 & \\
2 & 12.00 & & 17.33 & \\
3 & 17.67 & & 19.00 & \\
\hline
\end{tabular}

WISCI, Walking Index for Spinal Cord Injury; WISCI_i, WISCI-II initial; WICSI_6mo, WISCI-II after 6 months; SSEP, somatosensory evoked potentials; MEP, motor evoked potentials. 
Table 5. Linear multiple regression model on WISCI-II after 6 months in response to neurological, electrophysiological, and proprioception variables $(\mathrm{n}=14)$

\begin{tabular}{|c|c|c|c|c|}
\hline Variable & Corrected $^{2}$ & $\Delta \mathbf{p}$ & $\beta$ & p-value \\
\hline Model 1 & -0.178 & 0.833 & & \\
\hline LEMS & & & -0.365 & NS \\
\hline MEP & & & 0.778 & NS \\
\hline SSEP & & & -0.449 & NS \\
\hline Model 2 & 0.675 & $0.061^{*}$ & & \\
\hline LEMS & & & -0.240 & NS \\
\hline MEP & & & 0.559 & NS \\
\hline PRE & & & -0.997 & $0.020^{* *}$ \\
\hline Model 3 & 0.722 & $0.095^{*}$ & & \\
\hline TSI & & & -0.614 & NS \\
\hline LEMS & & & -0.278 & NS \\
\hline MEP & & & 1.090 & NS \\
\hline PRE & & & -0.475 & NS \\
\hline Model 4 & 0.754 & $0.080^{*}$ & & \\
\hline Type of injury & & & -2.068 & NS \\
\hline LEMS & & & -0.328 & NS \\
\hline MEP & & & 0.268 & NS \\
\hline PRE & & & -1.180 & $0.020^{* *}$ \\
\hline
\end{tabular}

WISCI, Walking Index for Spinal Cord Injury; LEMS, lower extremities motor score; MEP, motor-evoked potentials; SSEP, somatosensory evoked potentials; PRE, passive repositioning error; TSI, time since injury; type of injury (tetraplegia $=0$, paraplegia $=1$ ); NS, not significant.

${ }^{*} \mathrm{p}<0.1,{ }^{* *} \mathrm{p}<0.05$.

examination, but not with the WISCI 6 months later. Wirz et al. [23] reported that LEMS is not necessarily associated with improved locomotor function, and improvement in locomotor function does not always reflect an increase in LEMS. Extra-activation of the lower extremity muscles by the proprioception feedback during gait is one possible explanation for this discordance [24]. Our finding that proprioception measured with a dynamometer is related to the ambulatory capacity, supplements this hypothesis and emphasizes the importance of lower-extremity proprioception in ambulatory capacity in SCI patients.

In predicting the ambulatory capacity after 6 months, combining PRE with LEMS and MEP provided a better prediction of the ambulatory outcome, than combination of SSEP with LEMS and MEP. Since LEMS and SSEP/MEP are the clinical variables currently used for SCI patients, adding proprioception measurement using a dynamometer to these examinations might yield a more accurate prediction of the ambulatory capacity in the clinical setting.

One of the limitations of our study is that the subject group was confined to AIS D patients with various etiologies. The proprioception test in our study used both passive and active measurement in the bilateral knee joint, which takes more than 45 minutes to complete with each patient. Due to the time-consuming nature, it was performed only with patients who could sit in a chair for more than 45 minutes. Therefore, the patients in our study had relatively less severe SCI (AIS D), and our study results cannot be generalized to all SCI patients at this juncture. Future studies using an abridged protocol might be applicable to patients with more severe SCI (AIS B or C).

In conclusion, proprioception of the knee can be measured quantitatively with a dynamometer, and is related to the outcome of ambulatory capacity in incomplete SCI patients. Along with neurological and electrophysiological examinations, a passive repositioning knee proprioception test using a dynamometer may have supplemental value in predicting ambulatory capacity in incomplete SCI patients. 


\section{CONFLICT OF INTEREST}

No potential conflict of interest relevant to this article was reported.

\section{REFERENCES}

1. Curt A, Keck ME, Dietz V. Functional outcome following spinal cord injury: significance of motor-evoked potentials and ASIA scores. Arch Phys Med Rehabil 1998;79:81-6.

2. Curt A, Dietz V. Ambulatory capacity in spinal cord injury: significance of somatosensory evoked potentials and ASIA protocol in predicting outcome. Arch Phys Med Rehabil 1997;78:39-43.

3. Waters RL, Adkins RH, Yakura JS, Sie I. Motor and sensory recovery following incomplete tetraplegia. Arch Phys Med Rehabil 1994;75:306-11.

4. Burns SP, Golding DG, Rolle WA Jr, Graziani V, Ditunno JF Jr. Recovery of ambulation in motorincomplete tetraplegia. Arch Phys Med Rehabil 1997;78:1169-72.

5. Oleson CV, Burns AS, Ditunno JF, Geisler FH, Coleman WP. Prognostic value of pinprick preservation in motor complete, sensory incomplete spinal cord injury. Arch Phys Med Rehabil 2005;86:988-92.

6. Waters RL, Adkins R, Yakura J, Vigil D. Prediction of ambulatory performance based on motor scores derived from standards of the American Spinal Injury Association. Arch Phys Med Rehabil 1994;75:756-60.

7. Van Middendorp JJ, Hosman AJ, Pouw MH; EM-SCI Study Group, Van de Meent H. ASIA impairment scale conversion in traumatic SCI: is it related with the ability to walk? A descriptive comparison with functional ambulation outcome measures in 273 patients. Spinal Cord 2009;47:555-60.

8. Curt A, Dietz V. Electrophysiological recordings in patients with spinal cord injury: significance for predicting outcome. Spinal Cord 1999;37:157-65.

9. Pai YC, Rymer WZ, Chang RW, Sharma L. Effect of age and osteoarthritis on knee proprioception. Arthritis Rheum 1997;40:2260-5.

10. Barrett DS, Cobb AG, Bentley G. Joint proprioception in normal, osteoarthritic and replaced knees. J Bone Joint Surg Br 1991;73:53-6.

11. Nagai T, Sell TC, Abt JP, Lephart SM. Reliability, preci- sion, and gender differences in knee internal/external rotation proprioception measurements. Phys Ther Sport 2012;13:233-7.

12. Drouin JM, Valovich-mcLeod TC, Shultz SJ, Gansneder BM, Perrin DH. Eur J Appl Physiol 2004;91:22-9.

13. Kirshblum SC, Burns SP, Biering-Sorensen F, Donovan W, Graves DE, Jha A, et al. International standards for neurological classification of spinal cord injury (revised 2011). J Spinal Cord Med 2011;34:535-46.

14. DeLisa JA, MacKenzie K, Baran EM. Manual of nerve conduction velocity and somatosensory evoked potentials. 2nd ed. New York: Raven Press; 1987.

15. Di Lazzaro V, Oliviero A, Profice P, Ferrara L, Saturno E, Pilato F, et al. The diagnostic value of motor evoked potentials. Clin Neurophysiol 1999;110:1297-307.

16. Dittuno PL, Ditunno JF Jr. Walking index for spinal cord injury (WISCI II): scale revision. Spinal Cord 2001;39:654-6.

17. Lephart SM, Pincivero DM, Rozzi SL. Proprioception of the ankle and knee. Sports Med 1998;25:149-55.

18. Riskowski JL, Mikesky AE, Bahamonde RE, Alvey TV 3rd, Burr DB. Proprioception, gait kinematics, and rate of loading during walking: are they related? J Musculoskelet Neuronal Interact 2005;5:379-87.

19. Amatachaya S, Amatachaya P, Keawsutthi M, Siritaratiwat W. External cues benefit walking ability of ambulatory patients with spinal cord injury. J Spinal Cord Med 2013;36:638-44.

20. Bayramoglu M, Toprak R, Sozay S. Effects of osteoarthritis and fatigue on proprioception of the knee joint. Arch Phys Med Rehabil 2007;88:346-50.

21. Yang JM, Kim SY. Correlation of knee proprioception with muscle strength and spasticity in stroke patients. J Phys Ther Sci 2015;27:2705-8.

22. Arockiaraj J, Korula RJ, Oommen AT, Devasahayam S, Wankhar S, Velkumar S, et al. Proprioceptive changes in the contralateral knee joint following anterior cruciate injury. Bone Joint J 2013;95B:188-91.

23. Wirz M, van Hedel HJ, Rupp R, Curt A, Dietz V. Muscle force and gait performance: relationships after spinal cord injury. Arch Phys Med Rehabil 2006;87:1218-22.

24. Maegele M, Muller S, Wernig A, Edgerton VR, Harkema SJ. Recruitment of spinal motor pools during voluntary movements versus stepping after human spinal cord injury. J Neurotrauma 2002;19:1217-29. 